\title{
El club de lectura como metodología activa para mejorar las competencias profesionales de futuros periodistas
}

\author{
Amador Iranzo $^{a}$ y Miguel Ángel Fortea ${ }^{b}$ \\ a'Universitat Jaume I (Castelló), iranzo@uji.es; ${ }^{\text {bUniversitat Jaume I (Castelló), bfortea@uji.es }}$
}

\begin{abstract}
The purpose of this paper is to analyze the experience of the reading club that was developed in the second year of the Degree in Journalism of the Universitat Jaume I (Castello') during the academic year 2017-18. The activity, offered to students outside school hours, had as main objectives to encourage the habit of reading, to foster the capacity for reflection and the critical thinking and to improve the training of future journalists. The reading club was divided into two parts, linked to the two subjects of journalistic genres of the academic year, one of first quadrimester and another of the second. The reading club of the first quadrimester discussed works by authors such as Kapuścinsski and Caparrós, while in the second, the students had to write journalistic pieces following the analyzed texts as a model. In the subsequent survey, the participants evaluated the activity with a very high grade. In addition, the results showed that the reading club fulfilled the objectives that had been initially marked.
\end{abstract}

Keywords: reading club, Grade in Journalism, seminars (teaching method), learning methods, experiential learning, practical work (learning method)

\section{Resumen}

La presente comunicación tiene como objetivo analizar la experiencia del club de lectura que se desarrolló en el segundo curso del Grado en Periodismo de la Universitat Jaume I (Castelló) durante el curso 2017-18. La actividad, ofrecida a los estudiantes fuera del horario lectivo, tenía como objetivos principales incentivar el hábito de lectura, fomentar la capacidad de reflexión y el espíritu crítico y mejorar la formación de los futuros periodistas. El club de lectura se dividió en dos partes, vinculadas a las dos asignaturas de Géneros Periodísticos del curso, una de primer cuatrimestre y otra del segundo. El club de lectura del primer cuatrimestre debatió sobre obras de autores como Kapuściński y Caparrós, mientras que, en el del segundo, los estudiantes debían redactar piezas periodísticas siguiendo como modelo los textos analizados. En la encuesta posterior, los participantes evaluaron la actividad con una nota muy alta. Además, los resultados mostraron que el club de lectura cumplió con los objetivos que se habian marcado inicialmente.

Palabras clave: club de lectura, Grado en Periodismo, seminario (método pedagógico), método de aprendizaje, aprendizaje activo, trabajos prácticos

\section{Introducción}

La docencia universitaria debe fundamentarse, para dar respuesta al derecho general de todos los estudiantes universitarios establecido en el Real Decreto 1791/2010, de 30 de diciembre, por el que se aprueba el Estatuto del Estudiante Universitario, en el uso de metodologías activas de docencia y aprendizaje. En este sentido, Huber (2008), haciéndose eco de la propuesta de Shuell (1986), caracteriza 
las metodologías activas como un proceso de enseñanza/aprendizaje caracterizado por cinco rasgos: A) aprendizaje activo (cada estudiante tiene que aprender por sí mismo); B) aprendizaje autorregulado (los estudiantes tienen que evaluar los resultados de sus propias actividades por sí mismos); C) aprendizaje constructivo (los estudiantes construyen su propio conocimiento); D) aprendizaje situado (el contexto de aprendizaje permite oportunidades reales de aplicar los conocimientos adquiridos), y E) aprendizaje social (el proceso de enseñanza y aprendizaje es una interacción social).

De Miguel (2005), por su parte, establece una interesante distinción entre la metodología del proceso de enseñanza-aprendizaje y la modalidad de enseñanza. Esta última queda definida como «los distintos escenarios donde tienen lugar las actividades a realizar por el profesorado y el alumnado a lo largo de un curso, y que se diferencian entre sí en función de los propósitos de la acción didáctica» (De Miguel, 2005: 31). Entre las diferentes modalidades propuestas para una enseñanza centrada en el desarrollo de las competencias del estudiante, se propone el seminario como un «espacio físico o escenario donde se construye con profundidad una temática específica del conocimiento en el curso de su desarrollo y a través de intercambios personales entre los asistentes» (De Miguel, 2005: 56). La finalidad principal de esta modalidad de enseñanza es «construir conocimiento a través de la interacción y la actividad» (De Miguel, 2005: 34), por lo que esta forma de organizar la enseñanza va a facilitar sobremanera el uso de las metodologías activas que demanda la docencia universitaria centrada en competencias.

El seminario es una modalidad de organización del proceso de enseñanza-aprendizaje que se nutre de los métodos de enseñanza de socialización didáctica u orientados a la discusión y al trabajo en equipo (Fernández March, 2006), siendo habitual su combinación con otras metodologías de interacción grupal como los estudios de casos, el aprendizaje basado en problemas, etc. Pero, además, es conveniente que los temas tratados en los seminarios, así como sus materiales y recursos de apoyo, sean significativos, reales y relacionados con la profesión para favorecer el aprendizaje auténtico o situado (Brown, 2015; Díaz Barriga, 2006; Fortea, Sánchez-Tarazaga y Zorrilla, 2017).

La enseñanza a través de seminarios apenas se utilizaba en la docencia universitaria española de Licenciatura, mientras que, tras la implantación del crédito ECTS y la formación basada en competencias, son muchas las universidades que, como en el caso de la Universitat Jaume I, la incorporan en los diseños curriculares de la mayoría de sus estudios de grado. En estudios universitarios como los de Periodismo, que incorporan en el currículo competencias específicas profesionales basadas en la comunicación y la expresión escrita, un club de lectura constituye, además de una variante particular de seminario, una metodología activa de enseñanza auténtica. La propuesta de una lectura específica y común para todos los estudiantes participantes en el club, elegida estratégicamente con criterios pedagógicos, y la generación de un debate en torno al estilo de la redacción y otros aspectos formales (además de abordar los contenidos del escrito), facilita la discusión, la reflexión y la construcción compartida del conocimiento, conocimiento que en este caso tiene un claro carácter profesional aplicado. Los periodistas, en el ejercicio de su profesión, deben estar preparados para emitir sus juicios y opiniones y defenderlos ante posturas contrapuestas: el propio debate en sí, en grupos o tertulias públicas, es parte de un ejercicio real, y muy actual, de la profesión de periodista.

Este trabajo tiene como finalidad analizar una experiencia de innovación docente consistente en la implementación de un club de lectura sobre textos periodísticos. 


\section{Objetivos}

El objetivo general del presente trabajo es evaluar la eficacia de la metodología empleada en la presente experiencia, el seminario concretado en un club de lectura. El seminario es eficaz si a través de su desarrollo se logran los siguientes objetivos concretos:

- Conseguir que los alumnos se apunten por su propia motivación intrínseca (interés por su formación) en una actividad que se realiza fuera del horario académico.

- Que los estudiantes participantes estén satisfechos con la actividad y la perciban útil para su formación académica como futuros profesionales (desarrollo de competencias).

- Mejorar el rendimiento académico del estudiante participante en la experiencia de innovación.

\section{Desarrollo de la innovación}

El club de lectura se planteó como una actividad a desarrollar fuera del horario lectivo durante el segundo curso del Grado en Periodismo en la Universitat Jaume I (UJI) de Castelló. Esta experiencia docente nace como una iniciativa del Seminario Permanente de Innovación Educativa en Metodología de Enseñanza de Periodismo (SPIEMEP), el cual contó con la financiación de la convocatoria de ayudas a la innovación educativa de la UJI ${ }^{1}$. Esta comunicación relata la experiencia del club de lectura durante el curso académico 2017/18, segundo curso de implementación de la innovación. Para este segundo año de experiencia se plantearon los siguientes objetivos pedagógicos:

- Incentivar el hábito de lectura en los estudiantes, especialmente de textos periodísticos.

- Fomentar el espíritu crítico y la capacidad de reflexión en torno al mundo del periodismo.

- Mejorar la formación de los estudiantes, conectando la actividad docente con la práctica profesional.

El club de lectura se dividió en dos fases, vinculadas a dos asignaturas: Géneros Periodísticos I (de primer cuatrimestre) (GPI) y Géneros Periodísticos II (de segundo cuatrimestre) (GPII). Para fomentar la participación en el club de lectura del primer cuatrimestre, se premió a los alumnos con hasta medio punto adicional en la nota de la parte práctica de la asignatura. A este club de lectura asistieron 34 estudiantes (35,7\% de los 95 matriculados), un cifra que se consideró excesiva para trabajar, por lo que se optó por dividirlos en dos grupos. De cara al club del segundo cuatrimestre, se suprimió el incentivo en la nota y se limitó la participación a un máximo de 20 estudiantes. Finalmente, se apuntaron 24 alumnos, que fueron aceptados en su totalidad (21,8\% de los 110 matriculados en la asignatura).

El tema que articuló el club de lectura del primer cuatrimestre fue «Misión y retos del periodismo». Para abordarlo, se seleccionaron fragmentos de los libros Los cínicos no sirven para este oficio, de Ryszard Kapuściński (2002), y Lacrónica, de Martín Caparrós (2015), además de la carta al suscriptor número 34 (correspondiente al 17 de septiembre de 2017), escrita por Xosé Manuel Pereiro, de la publicación digital Contexto y Acción. Los estudiantes debían preparar una intervención inicial sobre la cuestión planteada y participar en el debate posterior. Después de la sesión presencial, se abrió un foro de discusión en el aula virtual, que permitió la interacción entre los participantes de los dos seminarios. Para facilitar el debate en línea, el profesor planteó un par de preguntas iniciales sobre el compromiso ético del periodismo y la vinculación entre periodismo y cultura, a las que se agregaron otras cuestiones formuladas por los propios estudiantes.

\footnotetext{
${ }^{1}$ Título del proyecto financiado: "Desarrollo de actividades dirigidas a incentivar los hábitos de lectura del estudiantado y la coordinación de las prácticas de las asignaturas del Grado en Periodismo". Código: 3434/17.
} 
El club de lectura del segundo cuatrimestre se planteó desde una perspectiva más práctica: los estudiantes, después de analizar los textos elegidos, debían redactar una pieza periodística — que opcionalmente podían presentar después como una de las prácticas obligatorias de la asignatura - tomando como referencia el estilo de los autores estudiados. El género periodístico que se trabajó en concreto durante el segundo cuatrimestre fue la crónica. De esta forma, se pretendía vincular la lectura y el estudio de textos de periodistas de referencia con el ejercicio profesional. De manera específica, los textos seleccionados pertenecían a los libros Lacrónica, de Martín Caparrós (2015); El nuevo periodismo, de Tom Wolfe (2012), y La suela de mis zapatos, de Gonzalo Suárez (2006). Los textos elegidos tenían el denominador común de poder asociarse a lo que se ha denominado nuevo periodismo, es decir, el uso de técnicas de la ficción en la redacción periodística. De esta forma, se ofrecía a los estudiantes una visión de la profesión diferente a la tradicional que se enseña en las aulas y se ejerce en los medios. El tema que se planteó para los crónicas que debían escribir los estudiantes fue las acciones de protesta.

\section{Resultados}

A continuación se presentan los resultados obtenidos con la encuesta anónima en línea preparada para evaluar la actividad una vez finalizada. Esta encuesta fue contestada por un total de 29 alumnos (50 \% del global de participantes), siendo 15 alumnos del club de lectura del primer cuatrimestre $(44,1 \%$ de los asistentes) y 14 del de segundo cuatrimestre $(58,3 \%)$.

\subsection{Motivación para participar en la experiencia de innovación}

Sobre las razones para participar en la actividad, el principal motivo señalado en el primer cuatrimestre fue la posibilidad de mejorar la nota en la asignatura, mientras que, en el segundo cuatrimestre, tras eliminar la posibilidad de obtener puntos adicionales en la calificación de la asignatura, el interés se deplazó hacia motivaciones más intrínsecas vinculadas con el aprendizaje como «profundizar en el conocimiento del periodismo»o «desarrollar competencias de la asignatura» como la redacción.

Tabla 1. Motivos de los estudiantes para participar en el seminario

\begin{tabular}{|c|c|c|c|c|c|c|}
\hline & \multicolumn{2}{|c|}{$\begin{array}{l}\text { Primer } \\
\text { cuatrimestre }\end{array}$} & \multicolumn{2}{|c|}{$\begin{array}{l}\text { Segundo } \\
\text { cuatrimestre }\end{array}$} & \multicolumn{2}{|c|}{ GLOBAL } \\
\hline & $\mathrm{N}$ & $\%$ & $\mathrm{~N}$ & $\%$ & $\mathrm{~N}$ & $\%$ \\
\hline Mejorar la nota en la asignatura & 7 & $46,7 \%$ & 0 & $0 \%$ & 7 & $24 \%$ \\
\hline Conocer nuevos autores y obras periodísticas & 4 & $26,7 \%$ & 4 & $28,5 \%$ & 8 & $28 \%$ \\
\hline Profundizar en el conocimiento del periodismo & 3 & $20 \%$ & 6 & $43 \%$ & 9 & $31 \%$ \\
\hline $\begin{array}{l}\text { Mejorar la redacción (competencias de la } \\
\text { asignatura) }\end{array}$ & 1 & $6,7 \%$ & 4 & $28,5 \%$ & 5 & $17 \%$ \\
\hline TOTAL & 15 & $100 \%$ & 14 & $100 \%$ & 29 & $100 \%$ \\
\hline
\end{tabular}




\subsection{Interés y utilidad de la experiencia para la formación académcia desde la perspectiva de los estudiantes.}

El nivel de satisfacción general con el club de lectura fue muy alto, con una puntuación media de 8 sobre 10 (equivalente a un «notable»), con una desviación típica de 1,2. En la tabla 2 se presenta el interés que suscitaron las diferentes actividades del seminario. Todas se situaron en la escala entre «bastante interesantes» y «muy interesantes», salvo el debate en línea (actividad que solo se realizó en el club de lectura del primer cuatrimestre).

Tabla 2. Interés de las actividades del seminario

\begin{tabular}{lccc}
\hline & Media & $\begin{array}{c}\text { Desviación } \\
\text { típica }\end{array}$ & $\mathrm{N}$ \\
\hline Las lecturas periodísticas & 2,36 & 0,62 & 29 \\
El debate presencial sobre las lecturas & 2,61 & 0,57 & 15 \\
El debate en línea sobre las lecturas & 1,67 & 0,72 & 14 \\
La redacción de la crónica & 2,21 & 0,58 & \\
\hline
\end{tabular}

(*) Escala de respuesta: 1 (nada interesante), 2 (bastante interesante) y 3 (muy interesante).

La tabla 3 resume las respuestas sobre el grado de acuerdo o desacuerdo de los estudiantes con diferentes afirmaciones vinculadas con la utilidad del club de lectura para su formación académica. En todos los aspectos se supera de media el nivel 3 de la escala («bastante de acuerdo»).

Tabla 3. Utilidad para la formación académica de los estudiantes

\begin{tabular}{lcc}
\hline & Media & $\begin{array}{c}\text { Desviación } \\
\text { típica }\end{array}$ \\
\hline $\begin{array}{l}\text { La actividad me ha servido para reflexionar y tener una visión más } \\
\text { crítica sobre el mundo del periodismo }\end{array}$ & 3,41 & 0,68 \\
$\begin{array}{l}\text { La actividad me ha servido para conocer nuevos autores y obras } \\
\text { periodísticas }\end{array}$ & 3,69 & 0,54 \\
$\begin{array}{l}\text { La actividad ha tenido un impacto positivo sobre mi formación } \\
\text { como periodista }\end{array}$ & 3,38 & 0,56 \\
$\begin{array}{l}\text { La actividad ha incrementado mi interés por leer textos } \\
\text { periodísticos }\end{array}$ & 3,07 & 29 \\
\hline
\end{tabular}

(*) Escala de respuesta: 0 (totalmente en desacuerdo), 1 (algo en desacuerdo), 3 (bastante de acuerdo) y 4 (totalmente de acuerdo).

Fuente: Elaboración propia a partir de los datos de la encuesta. 


\subsection{Resultados académicos de los estudiantes participantes}

Como se ve en la tabla 4, la nota media (excluida la puntuación adicional) en primera convocatoria de la parte práctica de la asignatura de los estudiantes que se apuntaron al club de lectura fue superior a la de los no participantes, con significación estadística en ambas asignaturas ( $\mathrm{T}$ de Student de 2,3 con una probabilidad $\mathrm{P}=.024$ para GP1 y $\mathrm{T}=2,42$ y $\mathrm{P}=.020$ para GPII). También fue superior la tasa de éxito, esto es, el porcentaje de alumnos aprobados respecto a los presentados).

Tabla 4. Rendimiento académico de los estudiantes que participan en el seminario

\begin{tabular}{|c|c|c|c|c|c|c|}
\hline & \multicolumn{2}{|c|}{$\begin{array}{c}\text { Estudiantes } \\
\text { participantes en el } \\
\text { seminario }\end{array}$} & \multicolumn{2}{|c|}{$\begin{array}{c}\text { Estudiantes que no } \\
\text { participan en el seminario }\end{array}$} & \multicolumn{2}{|c|}{$\begin{array}{c}\text { Global } \\
\text { de la Asignatura }\end{array}$} \\
\hline & $\begin{array}{l}\text { Nota } \\
\text { media } \\
(*)\end{array}$ & $\begin{array}{c}\% \\
\text { Aprobado } \\
(* *)\end{array}$ & $\begin{array}{l}\text { Nota } \\
\text { media }\end{array}$ & $\begin{array}{c}\% \\
\text { Aprobado }\end{array}$ & $\begin{array}{l}\text { Nota } \\
\text { media }\end{array}$ & $\begin{array}{c}\% \\
\text { Aprobado }\end{array}$ \\
\hline \multicolumn{7}{|l|}{ Géneros Periodísticos I } \\
\hline $\begin{array}{l}\text { Primer cuatrimestre } \\
(\mathrm{GPI})\end{array}$ & 5,8 & $76,4 \%$ & 5,1 & $60,7 \%$ & 5,4 & $68,8 \%$ \\
\hline \multicolumn{7}{|l|}{ Géneros Periodísticos II } \\
\hline $\begin{array}{l}\text { Segundo cuatrimestre } \\
\text { (GPII) }\end{array}$ & 6,2 & $70,8 \%$ & 5 & $55,3 \%$ & 5,5 & $61,4 \%$ \\
\hline
\end{tabular}

(*) En la nota media de las prácticas no se tienen en cuenta los puntos adicionales que se otorgaban en el primer seminario para que los datos sean comparables entre seminarios y con los estudiantes no participantes.

(**) El procentaje de aprobados se calcula sobre el total de presentados (90 en GPI y 96 en GPII, incluyéndose todos los alumnos participantes en los seminarios).

Fuente: Elaboración propia a partir de los datos de la encuesta.

Al comparar la media global en el expediente de la carrera entre los participantes en la experiencia y los no participantes, se comprobó que la gran mayoría de los alumnos con buenos expedientes participaron en la experiencia, lo que tiene una clara influencia sobre los resultados. En la tabla 5 se presentan los mismos resultados que en la tabla anterior, pero comparando el $50 \%$ de los alumnos con peor expediente de los participantes en el club de lectura, con otro grupo seleccionado al azar de no participantes con expedientes casi idénticos (en GPI son dos grupos de 17 alumnos cada uno, coincidiendo ambos con una media del expediente de 7,41 y una desviación típica de .29 y en GPII dos grupos de 12 alumnos cada uno coincidiendo con una media del expediente de 7,43 y desaviación típica de .36). Las diferencias no son estadísticamente significativas, seguramente por el escaso tamaño de las muestras, pero en todos los casos se apunta a que los participantes del seminario obtienen mejor rendimiento académico en la asignatura que los no participantes, a igualdad de expediente medio en la carrera. 
Tabla 5. Rendimiento académico de los estudiantes que participan en el seminario con peores expedientes

\begin{tabular}{|c|c|c|c|c|c|c|}
\hline & \multicolumn{2}{|c|}{$\begin{array}{c}\text { Estudiantes } \\
\text { participantes en el } \\
\text { seminario }\end{array}$} & \multicolumn{2}{|c|}{$\begin{array}{l}\text { Estudiantes que no } \\
\text { participan en el seminario }\end{array}$} & \multicolumn{2}{|c|}{$\begin{array}{c}\text { Global } \\
\text { de la Asignatura }\end{array}$} \\
\hline & $\begin{array}{l}\text { Nota } \\
\text { media } \\
(*)\end{array}$ & $\begin{array}{c}\% \\
\text { Aprobado } \\
(* *)\end{array}$ & $\begin{array}{l}\text { Nota } \\
\text { media }\end{array}$ & $\begin{array}{c}\% \\
\text { Aprobado }\end{array}$ & $\begin{array}{l}\text { Nota } \\
\text { media }\end{array}$ & $\begin{array}{c}\% \\
\text { Aprobado }\end{array}$ \\
\hline \multicolumn{7}{|l|}{ Géneros Periodísticos I } \\
\hline $\begin{array}{l}\text { Primer cuatrimestre } \\
(\mathrm{GPI})\end{array}$ & 5,33 & $64,7 \%$ & 4,82 & $64,7 \%$ & 5,80 & $64,7 \%$ \\
\hline \multicolumn{7}{|l|}{ Géneros Periodísticos II } \\
\hline $\begin{array}{l}\text { Segundo cuatrimestre } \\
\text { (GPII) }\end{array}$ & 5,09 & $66,7 \%$ & 4,40 & $50,0 \%$ & 4,75 & $58,3 \%$ \\
\hline
\end{tabular}

Fuente: Elaboración propia a partir de los datos de la encuesta.

\section{Conclusiones}

Uno de los primeros aspectos a analizar ha sido la motivación de los estudiantes para apuntarse a una actividad que, como la del club de lectura, se realizaba fuera del horario lectivo y les suponía, por lo tanto, un esfuerzo adicional. En este sentido, destaca el dato de que casi la mitad de las personas que participaron en el club de lectura del primer semestre lo hicieron por un motivo meramente utilitarista: subir la nota de la asignatura. Ofrecer un incentivo para la participación tenía el objetivo evidente de atraer a estudiantes que pudieran ser, en principio, reacios a involucrarse en un actividad que les iba a requerir una dedicación adicional sin ninguna recompensa aparente. Por lo contrario, también comportaba el riesgo de captar a alumnos exclusivamente interesados en la nota y que no iban implicarse realmente en la iniciativa. El incentivo cumplió con su objetivo a costa de elevar en exceso el número de participantes, lo que complicó la gestión de la actividad hasta el punto de tener que organizarse dos grupos del mismo seminario. No obstante, algunos estudiantes que se apuntaron solo para subir nota quedaron muy satisfechos con el club de lectura. Un comentario en la pregunta de la encuesta de respuesta libre lo explicaba así: «Quiero darte las gracias por la actividad, como ya he remarcado mi intención era solo subir nota pero gracias a ir encontré uno de mis referentes en el periodismo y en la vida, y del que estoy leyendo ahora todo lo que puedo (Kapuściński)». El alto número de alumnos que se apuntaron al club de lectura del segundo cuatrimestre a pesar de eliminarse el incentivo del suplemento a la nota, junto a opiniones como la señalada anteriormente, indican que la estrategia resultó acertada porque permitió atraer a un buen número estudiantes que no estaban interesados en la actividad y fidelizarlos para que continuaran en ella, de forma que cumplió el primero de los objetivos concretos perseguidos con la presente experiencia de innovación.

Por lo que respecta al interés suscitado por el seminario y sus correspondientes actividades, tal como cabría esperar en coherencia con un correcto uso de esta metodología didáctica, la actividad más interesante resultó ser el debate presencial sobre las lecturas, de forma que se logró conformar un espacio donde construir en profundidad la temática a través del intercambio e interacción entre los asistentes (de Miguel, 2005). El mismo alumno citado anteriormente lo explicaba así: «El debate también fue bastante interesante y aprendimos todos mucho del resto y de reflexionar nosotros mismos». Por contra, la única 
actividad que no funcionó suficientemente bien fue el intento de sustituir o ampliar la interacción presencial con la interacción no presencial a través de debates autogestionados por los estudiantes en el aula virtual. Varios estudiantes no le ven suficiente interés si el profesor no asume la dirección y dinamiza la participación (aunque se lleve a cabo dentro del aula virtual de la asignatura) o si no se produce el cara a cara entre ellos. Además, la mayoría de estudiantes están bastante o totalmente de acuerdo con que el seminario fue útil para su formación (en todos los aspectos planteados: conocer autores, reflexionar críticamente, incrementar su interés por leer texto periodísticos y formarse como periodistas), alcanzándose de este modo el segundo de los objetivos planteados en la experiencia.

Finalmente, cabe destacar que, cumpliéndose el tercero de los objetivos, los alumnos participantes en el club de lectura obtuvieron, de media, mejores calificaciones que el conjunto de su clase y mejores tasas de aprobado. Como suele ser frecuente en las experiencias de innovación educativa con carácter voluntario para el estudiante, son los mejores alumnos y más motivados los que se apuntaron al seminario. No obstante, independientemente de los posibles sesgos en el perfil del estudiante, comparando dos grupos de estudiantes con rendimiento equivalente en la titulación en general, se ha comprobado como los alumnos que participan en la experiencia de innovación también tienden a mostrar un rendimiento académico superior a sus homólogos que no han participado (aspecto que debería constatarse con muestras más grandes).

Por tanto, dado el logro de los objetivos planteados y demostrada la eficacia de la innovación experimentada, cabe concluir que experiencias como la presente deberían integrarse y consolidarse en la docencia oficial de la asignatura de forma obligatoria para todos los estudiantes matriculados. Sin duda, el seminario ha resultado un estrategia útil para llevar a cabo en la universidad una enseñanza auténtica para formar al estudiante en competencias profesionales (en la línea de propuestas como la de Fernández March, 2010).

\section{Referencias}

Brown, S. (2015). "La evaluación auténtica: el uso de la evaluación para ayudar a los estudiantes a aprender" en Relieve, 21 (2), art. M4.

$<$ http://dx.doi.org/10.7203/relieve.21.2.7674> [Consulta: 13 de febrero de 2020]

CAPARros, M. (2015). Lacrónica. Madrid: Círculo de Tiza.

De Miguel, M. (DIR.) (2005). Modalidades de enseñanza centradas en el desarrollo de competencias. Orientaciones para promover el cambio metodológico en el Espacio Europeo de Educación Superior. Oviedo: Ediciones de la Universidad de Oviedo.

Diaz BARriga, F. (2006). Enseñanza situada: vínculo entre la escuela y la vida. México D. F.: McGrawHill.

España. Real Decreto 1791/2010, de 30 de diciembre, por el que se aprueba el Estatuto del Estudiante Universitario. BOE, 31 de diciembre de 2010, núm. 318, pp. 109353-109380.

Fernandez March, A. (2006). "Metodologías activas para la formación de competencias" en Educatio Siglo XXI, 24 , pp. 35-56.

FERNANDEZ MARCH, A. (2010). "La evaluación orientada al aprendizaje en un modelo de formación por competencias en la educación universitaria” en Revista de docencia universitaria, 8 (1), pp. 11-34.

Fortea, M. A., SAnchez-Tarazaga, L. y Zorrilla, L. (2017). “Aprendizaje en entornos laborales y evaluación auténtica” en @tic. revista d'innovació educativa, 19, pp. 17-21.

$<$ http://dx.doi.org/10.7203/attic.19.11030> [Consulta: 13 de febrero de 2020] 
Huber, G. L. (2008). “Aprendizaje activo y metodologías educativas" en Revista de Educación, número extraordinario 2008, pp. 59-81.

KAPUSCINSKI, R. (2002). Los cínicos no sirven para este oficio. Sobre el buen periodismo. Barcelona: Anagrama. Pereiro, X. M. (2017). “Cartas al suscriptor. Número 34” en Contexto y Acción, 17 de septiembre de 2017.

SHuELl (1986). "Cognitive conceptions of learning” en Review of Educational Research, 56, pp. 411-436.

SuAREZ, G. (2006). La suela de mis zapatos. Barcelona: Seix Barral.

Wolfe, T. (2012). El nuevo periodismo. Barcelona: Anagrama. 